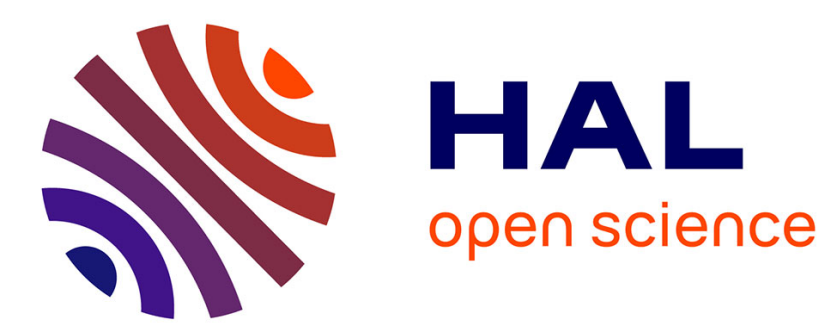

\title{
Extremal domains for the first eigenvalue of the Laplace-Beltrami operator
}

\author{
Frank Pacard, Pieralberto Sicbaldi
}

\section{To cite this version:}

Frank Pacard, Pieralberto Sicbaldi. Extremal domains for the first eigenvalue of the Laplace-Beltrami operator. Annales de l'Institut Fourier, 2009, 10.5802/aif.2438 . hal-01266405

\section{HAL Id: hal-01266405 \\ https://hal.science/hal-01266405}

Submitted on 3 Feb 2016

HAL is a multi-disciplinary open access archive for the deposit and dissemination of scientific research documents, whether they are published or not. The documents may come from teaching and research institutions in France or abroad, or from public or private research centers.
L'archive ouverte pluridisciplinaire HAL, est destinée au dépôt et à la diffusion de documents scientifiques de niveau recherche, publiés ou non, émanant des établissements d'enseignement et de recherche français ou étrangers, des laboratoires publics ou privés. 


\title{
EXTREMAL DOMAINS FOR THE FIRST EIGENVALUE OF THE LAPLACE-BELTRAMI OPERATOR
}

\author{
FRANK PACARD AND PIERALBERTO SICBALDI
}

\begin{abstract}
We prove the existence of extremal domains with small prescribed volume for the first eigenvalue of Laplace-Beltrami operator in some Riemannian manifold. These domains are close to geodesic spheres of small radius centered at a nondegenerate critical point of the scalar curvature.
\end{abstract}

\section{Statement of the Result}

Assume that we are given $(M, g)$ an $n$-dimensional Riemannian manifold. If $\Omega$ is a domain with smooth boundary in $M$, we denote by $\lambda_{\Omega}$ the first eigenvalue of $-\Delta_{g}$, the Laplace-Beltrami operator, in $\Omega$ with 0 Dirichlet boundary condition. A smooth domain $\Omega_{0} \subset M$ is said to be extremal if $\Omega \longmapsto \lambda_{\Omega}$ is critical at $\Omega_{0}$ with respect to variations of the domain $\Omega_{0}$ which preserve its volume. In order to make this definition precise, we first introduce the definition of deformation of $\Omega_{0}$.

Definition 1.1. We say that $\left\{\Omega_{t}\right\}_{t \in\left(-t_{0}, t_{0}\right)}$ is a deformation of $\Omega_{0}$, if there exists a vector field $\Xi$ such that $\Omega_{t}=\xi\left(t, \Omega_{0}\right)$ where $\xi(t, \cdot)$ is the flow associated to $\Xi$, namely

$$
\frac{d \xi}{d t}(t, p)=\Xi(\xi(t, p)) \quad \text { and } \quad \xi(0, p)=p .
$$

The deformation is said to be volume preserving if the volume of $\Omega_{t}$ does not depend on $t$.

If $\left\{\Omega_{t}\right\}_{t \in\left(-t_{0}, t_{0}\right)}$ is a domain deformation of $\Omega_{0}$, we denote by $\lambda_{t}$ the first eigenvalue of $-\Delta_{g}$ on $\Omega_{t}$, with 0 Dirichlet boundary conditions. Observe that both $t \longmapsto \lambda_{t}$ and the associated eigenfunction $t \longmapsto u_{t}$ (normalized to be positive and have $L^{2}\left(\Omega_{t}\right)$ norm equal to 1 ) inherits the regularity of the deformation of $\Omega_{0}$. These facts are standard and follow at once from the implicit function theorem together with the fact that the least eigenvalue of the Laplace-Beltrami operator with Dirichlet boundary condition is simple.

We can now give the definition of an extremal domain for the first eigenvalue of $-\Delta_{g}$ under Dirichlet boundary condition.

Definition 1.2. A domain $\Omega_{0}$ is an extremal domain for the first eigenvalue of $-\Delta_{g}$ if for any volume preserving deformation $\left\{\Omega_{t}\right\}_{t}$ of $\Omega_{0}$, we have

$$
\left.\frac{d \lambda_{t}}{d t}\right|_{t=0}=0
$$

where $\lambda_{t}$ is the first eigenvalue of $-\Delta_{g}$ on $\Omega_{t}$, with 0 Dirichlet boundary condition. 
For all $\epsilon>0$ small enough, we denote by $B_{\epsilon}(p) \subset M$ the geodesic ball of center $p \in M$ and radius $\epsilon$. We denote by $\stackrel{\circ}{\epsilon}_{\epsilon} \subset \mathbb{R}^{n}$ the Euclidean ball of radius $\epsilon$ centered at the origin.

Now we can state the main result of our paper :

Theorem 1.3. Assume that $p_{0}$ is a nondegenerate critical point of Scal, the scalar curvature function of $(M, g)$. Then, for all $\epsilon>0$ small enough, say $\epsilon \in\left(0, \epsilon_{0}\right)$, there exists a smooth domain $\Omega_{\epsilon} \subset M$ such that :

(i) The volume of $\Omega_{\epsilon}$ is equal to the Euclidean volume of $\stackrel{\circ}{\epsilon}_{\epsilon}$.

(ii) The domain $\Omega_{\epsilon}$ is extremal in the sense of definition 1.2.

Moreover, there exists $c>0$ and, for all $\epsilon \in\left(0, \epsilon_{0}\right)$, there exists $p_{\epsilon} \in M$ such that the boundary of $\Omega_{\epsilon}$ is a normal graph over $\partial B_{\epsilon}\left(p_{\epsilon}\right)$ for some function $w_{\epsilon}$ with

$$
\left\|w_{\epsilon}\right\|_{\mathcal{C}^{2, \alpha} \partial B_{\epsilon}\left(p_{\epsilon}\right)} \leq c \epsilon^{3} . \quad \text { and } \quad \operatorname{dist}\left(p_{\epsilon}, p_{0}\right) \leq c \epsilon .
$$

To put this result in perspective let us digress slightly and recall a few facts about the existence of constant mean curvature hypersurfaces in Riemannian manifolds. It is well known that solutions of the isoperimetric problem

$$
I_{\tau}:=\min _{\Omega \subset M: \operatorname{Vol} \Omega=\tau} \operatorname{Vol} \partial \Omega
$$

are (where they are smooth enough) constant mean curvature hypersurfaces. O. Druet [1] has proved that for small volumes (i.e. $\tau>0$ small), the solutions of the isoperimetric problem are close (in a sense to be made precise) to geodesic spheres of small radius centered at a point where the scalar curvature function on $(M, g)$ is maximal. Independently, R. Ye [13] has constructed constant mean curvature topological spheres which are close to geodesic spheres of small radius centered at a nondegenerate critical point of the scalar curvature function on $(M, g)$. Building on these results and a result of F. Pacard and X. Xu [10], S. Narduli [9] has obtained an asymptotic expansion of $I_{\tau}$ as $\tau$ tends to 0 .

It is well known ([4], [6], [7]) that the determination of the isoperimetric profile $I_{\tau}$ is related to the Faber-Krahn inequality where one looks for the least value of the first eigenvalue of the Laplace-Beltrami operator amongst domains with prescribed volume

$$
F K_{\tau}:=\min _{\Omega \subset M: \operatorname{Vol} \Omega=\tau} \lambda_{\Omega}
$$

Observe that a solution to this minimizing problem (when it is smooth) is an extremal domain in the sense of Definition 1.2. Therefore, Theorem 1.3 can be understood as a first step in understanding the asymptotics of $F K_{\tau}$ as $\tau$ is close to 0 .

Given the crucial rôle played by the critical points of the scalar curvature in the isoperimetric problem for small volumes, it is natural to expect that the critical points of the scalar curvature function will also be at the center of the study of $F K_{\tau}$ as $\tau$ is close to 0 and Theorem 1.3 is an illustration of such a link.

As a final remark, formal computations show that the estimate on $p_{\epsilon}$ can be improved into

$$
\operatorname{dist}\left(p_{\epsilon}, p_{0}\right) \leq c \epsilon^{2} \text {. }
$$


Since a rigorous proof of this estimate requires some extra technicalities which would have complicated the proof, we have chosen not to provide a proof of this fact.

\section{Preliminary Results}

The following well known result gives a formula for the first variation of the first eigenvalue for the Dirichlet problem under variations of the domain. This formula has been obtained by P. R. Garabedian and M. Schiffer in [3] when the underlying manifold is the euclidean space and by A. El Soufi and S. Ilias [2] (see Corollary 2.1) when the underlying manifold is a Riemannian manifold. For the sake of completeness we give here a proof based on arguments contained in a paper by D. Z. Zanger in [14] where a corresponding formula is derived for the Neumann problem.

Let $(M, g)$ be an $n$-dimensional Riemannian manifold. Assume that $\left\{\Omega_{t}\right\}_{t}$ is a perturbation of a domain $\Omega_{0}$ using the vector field $\Xi$, as defined in Definition 1.2. The outward unit normal vector field to $\partial \Omega_{t}$ is denoted by $\nu_{t}$. Let $u_{t} \in C^{2}\left(\Omega_{t}\right)$, be the corresponding smooth one-parameter family of Dirichlet first eigenfunctions of Laplace-Beltrami operator (normalized to be positive have $L^{2}\left(\Omega_{t}\right)$ norm equal to 1 ) with 0 Dirichlet boundary condition. The associated eigenvalue is denoted by $\lambda_{t}$.

We have the :

Proposition 2.1. [2] The derivative of $t \longmapsto \lambda_{t}$ at $t=0$ is given by

$$
\left.\frac{d \lambda_{t}}{d t}\right|_{t=0}=-\int_{\partial \Omega_{0}}\left(g\left(\nabla u_{0}, \nu_{0}\right)\right)^{2} g\left(\Xi, \nu_{0}\right) \mathrm{dvol}_{g},
$$

where $\mathrm{dvol}_{g}$ is the volume element on $\partial \Omega_{0}$ for the metric induced by $g$ and $\nu_{0}$ is the normal vector field about $\partial \Omega_{0}$.

Proof : We denote by $\xi$ the flow associated to $\Xi$. By definition, we have

$$
u_{t}(\xi(t, p))=0
$$

for all $p \in \partial \Omega_{0}$. Differentiating (1) with respect to $t$ and evaluating the result at $t=0$ we obtain

$$
\partial_{t} u_{0}=-g\left(\nabla u_{0}, \Xi\right) \text {, }
$$

on $\partial \Omega_{0}$. Now $u_{0} \equiv 0$ on $\partial \Omega_{0}$, and hence only the normal component of $\Xi$ plays a rôle in this formula. Therefore, we have

$$
\partial_{t} u_{0}=-g\left(\nabla u_{0}, \nu_{0}\right) g\left(\Xi, \nu_{0}\right),
$$

on $\partial \Omega_{0}$.

We differentiate with respect to $t$ the identity

$$
\Delta_{g} u_{t}+\lambda_{t} u_{t}=0 .
$$

and again evaluate the result at $t=0$. We obtain

$$
\Delta_{g} \partial_{t} u_{0}+\lambda_{0} \partial_{t} u_{0}=-\partial_{t} \lambda_{0} u_{0},
$$


in $\Omega_{0}$. Now we multiply (4) by $u_{0}$ and (3), evaluated the result at $t=0$, by $\partial_{t} u_{0}$, subtract the results and integrate it over $\Omega_{0}$ to get :

$$
\begin{aligned}
\partial_{t} \lambda_{0} \int_{\Omega_{0}} u_{0}^{2} \mathrm{dvol}_{g} & =\int_{\Omega_{0}}\left(\partial_{t} u_{0} \Delta_{g} u_{0}-u_{0} \Delta_{g} \partial_{t} u_{0}\right) \mathrm{dvol}_{g} \\
& =\int_{\partial \Omega_{0}}\left(\partial_{t} u_{0} g\left(\nabla u_{0}, \nu_{0}\right)-u_{0} g\left(\nabla \partial_{t} u_{0}, \nu_{0}\right)\right) \mathrm{dvol}_{g} \\
& =-\int_{\partial \Omega_{0}}\left(g\left(\nabla u_{0}, \nu_{0}\right)\right)^{2} g\left(\Xi, \nu_{0}\right) \operatorname{dvol}_{g},
\end{aligned}
$$

where we have used (2) and the fact that $u_{0}=0$ on $\partial \Omega_{0}$ to obtain the last equality. The result follows at once from the fact that $u_{0}$ is normalized to have $L^{2}\left(\Omega_{0}\right)$ norm equal to 1 .

This result allows us to state the problem of finding extremal domains into the solvability of an over-determined elliptic problem.

Proposition 2.2. A smooth domain $\Omega_{0}$ is extremal if and only if there exists a positive function $u_{0}$ and a constant $\lambda_{0}$ such that

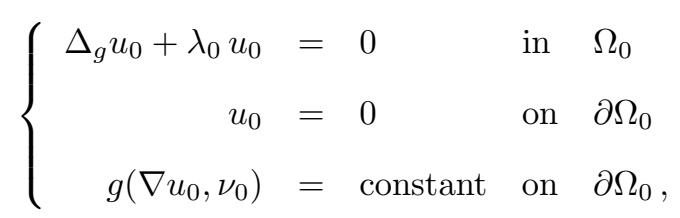

where $\nu_{0}$ is the normal vector field about $\partial \Omega_{0}$.

Proof : Assume that $u_{0}$ is a positive solution of (5). Observe that for a volume preserving variation, we have

$$
\int_{\partial \Omega_{0}} g\left(\Xi, \nu_{0}\right) \mathrm{dvol}_{g}=0
$$

Now, if $\lambda_{0}$ is a solution of (5), it is the first eigenvalue of $-\Delta_{g}$ on $\Omega_{0}$, under Dirichlet boundary condition. Moreover, we have

$$
\int_{\partial \Omega_{0}}\left(g\left(\nabla u_{0}, \nu_{0}\right)\right)^{2} g\left(\Xi, \nu_{0}\right) \mathrm{dvol}_{g}=0
$$

and the previous Proposition shows that the domain $\Omega_{0}$ is extremal in the sense of Definition 1.2.

Conversely, assume that $\Omega_{0}$ is extremal. Then given any function $w$ such that

$$
\int_{\partial \Omega_{0}} w \mathrm{dvol}_{g}=0
$$

it is easy to check that there exists a vector field $\Xi$ which generates a volume preserving deformation of $\Omega_{0}$ and which satisfies

$$
\Xi=w \nu_{0}
$$


EXTREMAL DOMAINS FOR THE FIRST EIGENVALUE OF THE LAPLACE-BELTRAMI OPERATOR 5

on $\partial \Omega_{0}$. The result of the previous Proposition implies that

$$
\int_{\partial \Omega_{0}}\left(g\left(\nabla u_{0}, \nu_{0}\right)\right)^{2} w \operatorname{dvol}_{g}=0 .
$$

The function $w$ being arbitrary, we conclude that $g\left(\nabla u_{0}, \nu_{0}\right)$ is a constant function and hence $u_{0}$ is a solution of (5). This completes the proof of the result.

Therefore, in order to find extremal domains, it is enough to find a domain $\Omega_{0}$ (regular enough) for which the over-determined problem (5) has a nontrivial positive solution. We will not be able

to solve this problem in full generality but we will be able to find solutions whose volumes are small.

\section{Rephrasing the PROBLEM}

To proceed, it will be useful to introduce the following notation. Given a point $p \in M$ we denote by $E_{1}, \ldots, E_{n}$ an orthonormal basis of the tangent plane to $M$ at $p$. Geodesic normal coordinates $x:=\left(x^{1}, \ldots, x^{n}\right) \in \mathbb{R}^{n}$ at $p$ are defined by

$$
X(x):=\operatorname{Exp}_{p}^{g}\left(\sum_{j=1}^{n} x^{j} E_{j}\right)
$$

We recall the Taylor expansion of the coefficients $g_{i j}$ of the metric $X^{*} g$ in these coordinates.

Proposition 3.1. At the point of coordinate $x$, the following expansion holds :

$$
g_{i j}=\delta_{i j}+\frac{1}{3} \sum_{k, \ell} R_{i k j \ell} x^{k} x^{\ell}+\frac{1}{6} \sum_{k, \ell, m} R_{i k j l, m} x^{k} x^{\ell} x^{m}+\mathcal{O}\left(|x|^{4}\right),
$$

Here $R$ is the curvature tensor of $g$ and

$$
\begin{aligned}
R_{i k j \ell} & =g\left(R\left(E_{i}, E_{k}\right) E_{j}, E_{\ell}\right) \\
R_{i k j \ell ; m} & =g\left(\nabla_{E_{m}} R\left(E_{i}, E_{k}\right) E_{j}, E_{\ell}\right),
\end{aligned}
$$

are evaluated at the point $p$.

The proof of this proposition can be found in [12], [8] or also in [11].

It will be convenient to identify $\mathbb{R}^{n}$ with $T_{p} M$ and $S^{n-1}$ with the unit sphere in $T_{p} M$. If $x:=\left(x^{1}, \ldots, x^{n}\right) \in \mathbb{R}^{n}$, we set

$$
\Theta(x):=\sum_{i=1}^{n} x^{i} E_{i} \in T_{p} M .
$$

Given a continuous function $f: S^{n-1} \longmapsto(0, \infty)$ whose $L^{\infty}$ norm is small (say less than the cut locus of $p$ ) we define

$$
B_{f}^{g}(p):=\left\{\operatorname{Exp}_{p}(\Theta(x)) \quad: \quad x \in \mathbb{R}^{n} \quad 0 \leq|x|<f(x /|x|)\right\} .
$$

The superscript $g$ is meant to remind the reader that this definition depends on the metric. 
Our aim is to show that, for all $\epsilon>0$ small enough, we can find a point $p \in M$ and a function $v: S^{n-1} \longrightarrow \mathbb{R}$ such that

$$
\operatorname{Vol} B_{\epsilon(1+v)}^{g}(p)=\epsilon^{n} \operatorname{Vol} \stackrel{\circ}{B}_{1}
$$

and the over-determined problem

$$
\left\{\begin{array}{rlrll}
\Delta_{g} \phi+\lambda \phi & = & 0 & \text { in } & B_{\epsilon(1+v)}^{g}(p) \\
\phi & = & 0 & \text { on } & \partial B_{\epsilon(1+v)}^{g}(p) \\
g(\nabla \phi, \nu) & = & \text { constant } & \text { on } & \partial B_{\epsilon(1+v)}^{g}(p)
\end{array}\right.
$$

has a nontrivial positive solution, where $\nu$ is the normal vector field about $\partial B_{\epsilon(1+v)}^{g}(p)$.

Observe that, considering the dilated metric $\bar{g}:=\epsilon^{-2} g$, the above problem is equivalent to finding a point $p \in M$ and a function $v: S^{n-1} \longrightarrow \mathbb{R}$ such that

$$
\operatorname{Vol} B_{1+v}^{\bar{g}}(p)=\operatorname{Vol} \stackrel{\circ}{B}_{1}
$$

and for which the over-determined problem

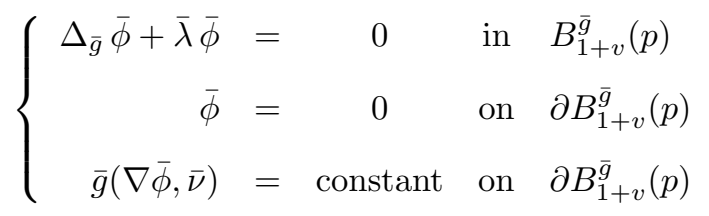

has a nontrivial positive solution, where $\bar{\nu}$ is the normal vector field about $\partial B_{1+v}^{\bar{g}}(p)$. The relation between the solutions of the two problems is simply given by

$$
\phi=\epsilon^{-n / 2} \bar{\phi}
$$

and

$$
\lambda=\epsilon^{-2} \bar{\lambda} .
$$

Let us denote by $\stackrel{\circ}{g}$ the Euclidean metric in $\mathbb{R}^{n}$ and $\lambda_{1}$ the first eigenvalue of $-\Delta_{g}$ in the unit ball $\stackrel{\circ}{B}_{1}$ with 0 Dirichlet boundary condition. We denote by $\phi_{1}$ the associated eigenfunction

$$
\left\{\begin{array}{rllll}
\Delta_{\grave{g}} \phi_{1}+\lambda_{1} \phi_{1} & = & 0 & \text { in } & \stackrel{\circ}{B}_{1} \\
\phi_{1} & = & 0 & \text { on } & \partial \dot{\circ}_{1}
\end{array} .\right.
$$

which is normalized to be positive and have $L^{2}\left(\stackrel{\circ}{B}_{1}\right)$ norm equal to 1 .

For notational convenience, given a continuous function $f: S^{n-1} \longmapsto(0, \infty)$, we set

$$
\stackrel{\circ}{B}_{f}:=\left\{x \in \mathbb{R}^{n} \quad: \quad 0 \leq|x|<f(x /|x|)\right\} .
$$

The following result follows from the implicit function theorem. 
Proposition 3.2. Given a point $p \in M$, there exists $\epsilon_{0}>0$ and for all $\epsilon \in\left(0, \epsilon_{0}\right)$ and all function $\bar{v} \in C^{2, \alpha}\left(S^{n-1}\right)$ satisfying

and

$$
\|\bar{v}\|_{\mathcal{C}^{2, \alpha}\left(S^{n-1}\right)} \leq \epsilon_{0}
$$

$$
\int_{S^{n-1}} \bar{v} \operatorname{dvol}_{\grave{g}}=0
$$

there exists a unique positive function $\bar{\phi}=\bar{\phi}(\epsilon, p, \bar{v}) \in \mathcal{C}^{2, \alpha}\left(B_{1+v}^{\bar{g}}(p)\right)$, a constant $\bar{\lambda}=\bar{\lambda}(\epsilon, p, \bar{v}) \in \mathbb{R}$ and a constant $v_{0}=v_{0}(\epsilon, p, \bar{v}) \in \mathbb{R}$ such that

$$
\operatorname{Vol}_{\bar{g}}\left(B_{1+v}\right)=\operatorname{Vol}_{\grave{g}}\left(\stackrel{\circ}{B}_{1}\right)
$$

where $v:=v_{0}+\bar{v}$ and $\bar{\phi}$ is a solution to the problem

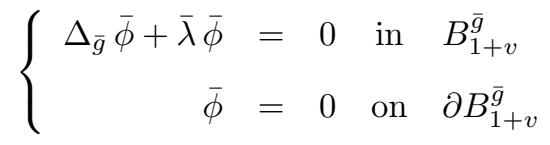

which is normalized by

$$
\int_{B_{1+v}^{\bar{g}}(p)} \bar{\phi}^{2} d v o l_{\bar{g}}=1
$$

In addition $\bar{\phi}, \bar{\lambda}$ and $v_{0}$ depend smoothly on the function $\bar{v}$ and the parameter $\epsilon$ and $\bar{\phi}=\phi_{1}$, $\bar{\lambda}=\lambda_{1}$ and $v_{0}=0$ when $\epsilon=0$ and $\bar{v} \equiv 0$.

Proof : Instead of working on a domain depending on the function $v=v_{0}+\bar{v}$, it will be more convenient to work on a fixed domain

$$
\stackrel{\circ}{B}_{1}:=\left\{y \in \mathbb{R}^{n} \quad: \quad|y|<1\right\}
$$

endowed with a metric depending on both $\epsilon$ and the function $v$. This can be achieved by considering the parameterization of $B_{1+v}^{\bar{g}}\left(=B_{\epsilon(1+v)}^{g}\right)$ given by

$$
Y(y):=\operatorname{Exp}_{p}^{\bar{g}}\left(\left(1+v_{0}+\chi(y) \bar{v}\left(\frac{y}{|y|}\right)\right) \sum_{i} y^{i} E_{i}\right)
$$

where $\chi$ is a cutoff function identically equal to 0 when $|y| \leq 1 / 2$ and identically equal to 1 when $|y| \geq 3 / 4$.

Hence the coordinates we consider from now on are $y \in \stackrel{\circ}{B}_{1}$ and in these coordinates the metric $\hat{g}:=Y^{*} \bar{g}$ can be written as

$$
\hat{g}=\left(1+v_{0}\right)^{2}\left(\stackrel{\circ}{g}+\sum_{i, j} C^{i j} d y_{i} d y_{j}\right),
$$

where the coefficients $C^{i j} \in \mathcal{C}^{1, \alpha}\left(\stackrel{\circ}{B}_{1}\right)$ are functions of $y$ depending on $\epsilon, v=v_{0}+\bar{v}$ and the first partial derivatives of $v$. Moreover, $C^{i j} \equiv 0$ when $\epsilon=0$ and $\bar{v}=0$.

Observe that

$$
\left(\epsilon, v_{0}, \bar{v}\right) \longmapsto C^{i j}(\epsilon, v)
$$


are smooth maps.

Up to some multiplicative constant, the problem we want to solve can now be rewritten in the form

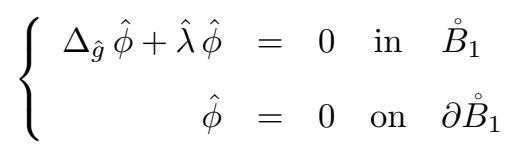

with

$$
\int_{\dot{B}_{1}} \hat{\phi}^{2} \operatorname{dvol}_{\hat{g}}=1
$$

and

$$
\operatorname{Vol}_{\hat{g}}\left(\stackrel{\circ}{B}_{1}\right)=\operatorname{Vol}_{\grave{g}}\left(\stackrel{\circ}{B}_{1}\right)
$$

When $\epsilon=0$ and $\bar{v} \equiv 0$, the metric $\hat{g}=\left(1+v_{0}\right)^{2} \stackrel{\circ}{g}$ is nothing but the Euclidean metric and a solution of (9) is therefore given by $\hat{\phi}=\phi_{1}, \hat{\lambda}=\lambda_{1}$ and $v_{0}=0$. In the general case, the relation between the function $\bar{\phi}$ in the statement of the Proposition and the function $\hat{\phi}$ is simply given by

$$
Y^{*} \bar{\phi}=\hat{\phi} \quad \text { and } \quad \bar{\lambda}=\hat{\lambda}
$$

For all $\psi \in \mathcal{C}^{2, \alpha}\left(\stackrel{\circ}{B}_{1}\right)$ such that

$$
\int_{\check{B}_{1}} \psi \phi_{1} \mathrm{dvol}_{\stackrel{g}{g}}=0
$$

we define

$$
N\left(\epsilon, \bar{v}, \psi, v_{0}\right):=\left(\Delta_{\grave{g}} \psi+\lambda_{1} \psi+\left(\Delta_{\hat{g}}-\Delta_{\grave{g}}+\mu\right)\left(\phi_{1}+\psi\right), \operatorname{Vol}_{\hat{g}}\left(\stackrel{\circ}{B}_{1}\right)-\operatorname{Vol}_{\grave{g}}\left(\stackrel{\circ}{B}_{1}\right)\right)
$$

where $\mu$ is given by

$$
\mu=-\int_{\dot{B}_{1}} \phi_{1}\left(\Delta_{\hat{g}}-\Delta_{\grave{g}}\right)\left(\phi_{1}+\psi\right) \mathrm{dvol}_{\grave{g}}
$$

so that the first entry of $M$ is $L^{2}\left(\stackrel{\circ}{B}_{1}\right)$-orthogonal to $\phi_{1}$. Observe that $N$ also depends on the choice of the point $p \in M$.

We have

$$
N(0,0,0,0)=(0,0) \text {. }
$$

It should be clear that the mapping $N$ is a smooth map from a neighborhood of $(0,0,0,0)$ in $[0, \infty) \times \mathcal{C}_{m}^{2, \alpha}\left(S^{n-1}\right) \times \mathcal{C}_{\perp, 0}^{2, \alpha}\left(\stackrel{\circ}{B}_{1}\right) \times \mathbb{R}$ into a neighborhood of $(0,0)$ in $\mathcal{C}_{\perp}^{0, \alpha}\left(\stackrel{\circ}{B}_{1}\right) \times \mathbb{R}$. Here the subscript $\perp$ indicates that the functions in the corresponding space are $L^{2}\left(\stackrel{\circ}{B}_{1}\right)$-orthogonal to $\phi_{1}$ (for the Euclidean metric) and the subscript 0 indicates that the functions vanish on the boundary of ${ }^{\circ}{ }_{1}$. Finally, the subscript $m$ indicates that the functions have mean 0 over the unit (Euclidean) sphere.

We claim that the partial differential of $N$ with respect to $\psi$, computed at $(0,0,0,0)$, is given by

$$
D_{\psi} N(0,0,0,0)=\left(\Delta_{g}+\lambda_{1}, 0\right)
$$


while the partial differential of $N$ with respect to $v_{0}$, computed at $(0,0,0,0)$, is given by

$$
\partial_{v_{0}} N(0,0,0,0)=\left(0, n \operatorname{Vol}_{\grave{g}}\left(\stackrel{\circ}{B}_{1}\right)\right)
$$

There is no difficulty in getting the expression of the first partial differential since $\hat{g}=\stackrel{\circ}{g}$ when $\epsilon=v_{0}=0$ and $\bar{v}=0$ and hence

$$
N(0,0, \psi, 0)=\left(\Delta_{\grave{g}} \psi+\lambda_{1} \psi+\mu\left(\phi_{1}+\psi\right), 0\right)
$$

where $\mu=0$. The derivation of the partial differential with respect to $v_{0}$ is not hard either but requires some care. Indeed, this time we have $\hat{g}=\left(1+v_{0}\right)^{2} \stackrel{\circ}{g}$ since $\bar{v} \equiv 0$ and $\epsilon=0$ and hence

$$
\begin{aligned}
N\left(0,0,0, v_{0}\right) & =\left(\left(\left(\left(1+v_{0}\right)^{-2}-1\right) \Delta_{\grave{g}}+\mu\right) \phi_{1},\left(\left(1+v_{0}\right)^{n}-1\right) \operatorname{Vol}_{\grave{g}}\left(\stackrel{\circ}{B}_{1}\right)\right) \\
& =\left(\left(\mu-\lambda_{1}\left(\left(1+v_{0}\right)^{-2}-1\right)\right) \phi_{1},\left(\left(1+v_{0}\right)^{n}-1\right) \operatorname{Vol}_{\grave{g}}\left(\stackrel{\circ}{B}_{1}\right)\right)
\end{aligned}
$$

where $\mu$ is given by

$$
\mu=-\left(\left(1+v_{0}\right)^{-2}-1\right) \int_{\dot{B}_{1}} \phi_{1} \Delta_{\grave{g}} \phi_{1} \operatorname{dvol}_{\grave{g}}=\lambda_{1}\left(\left(1+v_{0}\right)^{-2}-1\right) .
$$

So we get

$$
\partial_{v_{0}} N(0,0,0,0)=\left(\left(\left.\partial_{v_{0}} \mu\right|_{v_{0}=0}+2 \lambda_{1}\right) \phi_{1}, n \operatorname{Vol}_{\grave{g}}\left(\stackrel{\circ}{B}_{1}\right)\right)
$$

and

$$
\left.\partial_{v_{0}} \mu\right|_{v_{0}=0}=-2 \lambda_{1}
$$

The claim then follows at once.

Hence the partial differential of $N$ with respect to both $\psi$ and $v_{0}$, computed at $(0,0,0,0)$ is precisely invertible from $\mathcal{C}_{\perp, 0}^{2, \alpha}\left(\stackrel{\circ}{B}_{1}\right) \times \mathbb{R}$ into $\mathcal{C}_{\perp}^{0, \alpha}\left(\stackrel{\circ}{B}_{1}\right) \times \mathbb{R}$ and the implicit function theorem ensures, for all $(\epsilon, \bar{v})$ in a neighborhood of $(0,0)$ in $[0, \infty) \times \mathcal{C}_{m}^{2, \alpha}\left(S^{n-1}\right)$, the existence of a (unique) $\left(\psi, v_{0}\right) \in \mathcal{C}_{\perp, 0}^{2, \alpha}\left(\stackrel{\circ}{B}_{1}\right) \times \mathbb{R}$ such that $N\left(\epsilon, \bar{v}, \psi, v_{0}\right)=0$. The function $\hat{\phi}:=\phi_{1}+\psi$ solves $(12)$ and in order to have (13) fulfilled, it is enough to divide it by its $L^{2}$ norm. The fact that the solution depends smoothly on the parameter $\epsilon$, the function $\bar{v}$ and the point $p \in M$ is standard. This completes the proof of the result.

After canonical identification of $\partial B_{1+v}^{\bar{g}}(p)$ with $S^{n-1}$, we define, the operator $F$ :

$$
F(p, \epsilon, \bar{v})=\left.\bar{g}(\nabla \bar{\phi}, \bar{\nu})\right|_{\partial B_{1+v}^{\bar{g}}}-\frac{1}{\operatorname{Vol}_{\bar{g}}\left(\partial B_{1+v}^{\bar{g}}\right)} \int_{\partial B_{1+v}^{\bar{g}}} \bar{g}(\nabla \bar{\phi}, \bar{\nu}) \mathrm{dvol}_{\bar{g}},
$$

where $\bar{\nu}$ denotes the unit normal vector field to $\partial B_{1+v}^{\bar{g}}$ and $\left(\bar{\phi}, v_{0}\right)$ is the solution of $(10)$ provided by the previous result. Recall that $v=v_{0}+\bar{v}$. Schauder's estimates imply that $F$ is well defined from a neighborhood of $M \times(0,0)$ in $M \times[0, \infty) \times \mathcal{C}_{m}^{2, \alpha}\left(S^{n-1}\right)$ into $\mathcal{C}_{m}^{1, \alpha}\left(S^{n-1}\right)$. The subscript $m$ is meant to point out that the functions have mean 0 . Our aim is to find $(p, \epsilon, \bar{v})$ such that $F(p, \epsilon, \bar{v})=0$. Observe that, with this condition, $\bar{\phi}$ will be the solution to the problem (8). 
Following the proof of the previous result, we have the alternative expression for $F$.

$$
F(p, \epsilon, \bar{v})=\left.\hat{g}(\nabla \hat{\phi}, \hat{\nu})\right|_{\partial \dot{B}_{1}}-\frac{1}{\operatorname{Vol}_{\hat{g}}\left(\partial \dot{B}_{1}\right)} \int_{\partial \dot{B}_{1}} \hat{g}(\nabla \hat{\phi}, \hat{\nu}) \operatorname{dvol}_{\hat{g}}
$$

where this time $\hat{\nu}$ is the the unit normal vector field to $\partial \stackrel{\circ}{B}_{1}$ using the metric $\hat{g}$.

We end this section by the proof of the :

Lemma 3.3. There exists a constant $c>0$ such that, for all $p \in M$ and all $\epsilon \geq 0$ small enough we have

$$
\|F(p, \epsilon, 0)\|_{\mathcal{C}^{1, \alpha}} \leq c \epsilon^{2}
$$

For all $a \in \mathbb{R}^{n}$, the following estimate holds

$$
\left|\int_{S^{n-1}} \stackrel{\circ}{g}(a, \cdot) F(p, \epsilon, 0) \mathrm{dvol}_{g}-C \epsilon^{3} g(\nabla \operatorname{Scal}(p), \Theta(a))\right| \leq c \epsilon^{4}\|a\|,
$$

where

$$
C:=\frac{1}{2 n(n+2)} \frac{1}{\partial_{r} \phi_{1}(1)} \int_{\dot{B}_{1}} r^{2}\left|\partial_{r} \phi_{1}\right|^{2} \operatorname{dvol}_{\grave{g}}
$$

Proof : We keep the notations of the proof of the previous result with $\bar{v} \equiv 0$. In order to prove these estimates, we follow the construction of $F(p, \epsilon, 0)$ step by step. First of all, since $\bar{v} \equiv 0$, we have

and

$$
N(\epsilon, 0,0,0)=\left(\left(\Delta_{\hat{g}}-\Delta_{\grave{g}}+\mu\right) \phi_{1}, \operatorname{Vol}_{\hat{g}}\left(\stackrel{\circ}{B}_{1}\right)-\operatorname{Vol}_{\grave{g}}\left(\stackrel{\circ}{B}_{1}\right)\right),
$$

$$
\mu=-\int_{\dot{B}_{1}} \phi_{1}\left(\Delta_{\hat{g}}-\Delta_{\grave{g}}\right) \phi_{1} \mathrm{dvol}_{\grave{g}} .
$$

If in addition $v_{0}=0$, we can estimate

$$
\hat{g}_{i j}=\delta_{i j}+\mathcal{O}\left(\epsilon^{2}\right)
$$

hence

$$
N(\epsilon, 0,0,0)=\mathcal{O}\left(\epsilon^{2}\right)
$$

The implicit function theorem immediately implies that the solution of

$$
N\left(\epsilon, 0, \psi, v_{0}\right)=0
$$

satisfies

$$
\|\psi(\epsilon, p, 0)\|_{\mathcal{C}^{2, \alpha}}+\left|v_{0}(\epsilon, p, 0)\right| \leq c \epsilon^{2}
$$

To complete the proof, observe that $\hat{\nu}=\left(1+v_{0}\right)^{-1} \partial_{r}$ when $\bar{v} \equiv 0$. Therefore

$$
\hat{g}(\nabla \hat{\phi}, \hat{\nu})=\partial_{r} \phi_{1}+\mathcal{O}\left(\epsilon^{2}\right)
$$

(be careful that $\hat{g}$ is defined with $v_{0}=v_{0}(\epsilon, p, 0)$ and $\left.\bar{v} \equiv 0\right)$. Since $\partial_{r} \phi_{1}$ is constant along $\partial \dot{B}_{1}$, we conclude that

$$
F(p, \epsilon, 0)=\mathcal{O}\left(\epsilon^{2}\right)
$$

and this proves the first estimate. 
We now turn to the proof of the second estimate. Instead of going through the construction of $\hat{\phi}$ step by step, we compute

$$
\begin{aligned}
& \int_{S^{n-1}} \stackrel{g}{g}\left(\nabla \phi_{1}, a\right) \frac{\partial \hat{\phi}}{\partial r} \mathrm{dvol}_{\grave{g}}=\int_{\dot{B}_{1}} \stackrel{\circ}{g}\left(\nabla \phi_{1}, a\right)\left(\Delta_{\grave{g}}+\lambda_{1}\right) \hat{\phi} \mathrm{dvol}_{\grave{g}}-\int_{\dot{B}_{1}} \hat{\phi}\left(\Delta_{\grave{g}}+\lambda_{1}\right) \stackrel{g}{g}\left(\nabla \phi_{1}, a\right) \mathrm{dvol}_{\grave{g}} \\
& =\int_{\dot{B}_{1}} \stackrel{\circ}{g}\left(\nabla \phi_{1}, a\right)\left(\Delta_{\grave{g}}+\lambda_{1}\right) \hat{\phi} \mathrm{dvol}_{\grave{g}} \\
& =\int_{\dot{B}_{1}} \stackrel{\circ}{g}\left(\nabla \phi_{1}, a\right)\left(\Delta_{\grave{g}}-\Delta_{\hat{g}}\right) \hat{\phi} \mathrm{dvol}_{\grave{g}}+\left(\lambda_{1}-\hat{\lambda}\right) \int_{\dot{B}_{1}} \stackrel{\circ}{g}\left(\nabla \phi_{1}, a\right) \hat{\phi} \mathrm{dvol}_{\grave{g}} \\
& =\int_{\dot{B}_{1}} \stackrel{g}{g}\left(\nabla \phi_{1}, a\right)\left(\Delta_{\grave{g}}-\Delta_{\hat{g}}\right) \phi_{1} \operatorname{dvol}_{\grave{g}}+\left(\lambda_{1}-\hat{\lambda}\right) \int_{\dot{B}_{1}} \stackrel{\circ}{g}\left(\nabla \phi_{1}, a\right) \phi_{1} \mathrm{dvol}_{\grave{g}} \\
& +\int_{\dot{B}_{1}} \stackrel{\circ}{g}\left(\nabla \phi_{1}, a\right)\left(\Delta_{\grave{g}}-\Delta_{\hat{g}}\right)\left(\hat{\phi}-\phi_{1}\right) \mathrm{dvol}_{\grave{g}} \\
& +\left(\lambda_{1}-\hat{\lambda}\right) \int_{\dot{B}_{1}}\left(\nabla \phi_{1} \cdot a\right)\left(\hat{\phi}-\phi_{1}\right) \mathrm{dvol}_{\grave{g}} \\
& =\int_{\dot{B}_{1}} \stackrel{\circ}{g}\left(\nabla \phi_{1}, a\right)\left(\Delta_{\grave{g}}-\Delta_{\hat{g}}\right) \phi_{1} \mathrm{dvol}_{\grave{g}} \\
& +\int_{\dot{B}_{1}} \stackrel{\circ}{g}\left(\nabla \phi_{1}, a\right)\left(\Delta_{\grave{g}}-\Delta_{\hat{g}}\right)\left(\hat{\phi}-\phi_{1}\right) \mathrm{dvol}_{\grave{g}} \\
& +\left(\lambda_{1}-\hat{\lambda}\right) \int_{\dot{B}_{1}} \stackrel{\circ}{g}\left(\nabla \phi_{1}, a\right)\left(\hat{\phi}-\phi_{1}\right) \mathrm{dvol}_{\grave{g}}
\end{aligned}
$$

The last two terms can be estimated easily since $\hat{\lambda}-\lambda_{1}=\mathcal{O}\left(\epsilon^{2}\right), \hat{\phi}-\phi_{1}=\mathcal{O}\left(\epsilon^{2}\right)$ and the coefficients of $\Delta_{\hat{g}}-\Delta_{\hat{g}}$ are bounded by a constant times $\epsilon^{2}$. Therefore, we conclude that there exists a constant $c$ such that

$$
\left|\int_{S^{n-1}} \stackrel{g}{g}\left(\nabla \phi_{1}, a\right) \frac{\partial \hat{\phi}}{\partial r} \operatorname{dvol}_{\grave{g}}-\int_{\dot{B}_{1}} \stackrel{\circ}{g}\left(\nabla \phi_{1}, a\right)\left(\Delta_{\grave{g}}-\Delta_{\hat{g}}\right) \phi_{1} \operatorname{dvol}_{\grave{g}}\right| \leq c \epsilon^{4}\|a\|
$$

To proceed, we use the result of Proposition 3.1 to show that the coefficients of the metric $\hat{g}$ can be expanded as

$\hat{g}_{i j}(y)=\left(1+v_{0}\right)^{2}\left(\delta_{i j}+\frac{1}{3} \sum_{k, \ell} R_{i k j \ell} y^{k} y^{\ell}\left(1+v_{0}\right)^{2} \epsilon^{2}+\frac{1}{6} \sum_{k, \ell, m} R_{i k j l, m} y^{k} y^{\ell} y^{m}\left(1+v_{0}\right)^{3} \epsilon^{3}+\mathcal{O}\left(\epsilon^{4}\right)\right)$

Keeping in mind that $v_{0}=\mathcal{O}\left(\epsilon^{2}\right)$, this simplifies slightly into

$$
\hat{g}_{i j}(y)=\left(1+v_{0}\right)^{2}\left(\delta_{i j}+\frac{1}{3} \sum_{k, \ell} R_{i k j \ell} y^{k} y^{\ell} \epsilon^{2}+\frac{1}{6} \sum_{k, \ell, m} R_{i k j l, m} y^{k} y^{\ell} y^{m} \epsilon^{3}+\mathcal{O}\left(\epsilon^{4}\right)\right)
$$


This implies that

$$
\begin{aligned}
\hat{g}^{i j} & =\left(1+v_{0}\right)^{-2}\left(\delta_{i j}-\frac{1}{3} R_{i k j \ell} y^{k} y^{\ell} \epsilon^{2}-\frac{1}{6} R_{i k j \ell, m} y^{k} y^{\ell} y^{m} \epsilon^{3}\right)+\mathcal{O}\left(\epsilon^{4}\right) \\
\log |\hat{g}| & =2 n \log \left(1+v_{0}\right)+\frac{1}{3} R_{k \ell} y^{k} y^{\ell} \epsilon^{2}+\frac{1}{6} R_{k \ell, m} y^{k} y^{\ell} y^{m} \epsilon^{3}+\mathcal{O}\left(\epsilon^{4}\right)
\end{aligned}
$$

where

$$
R_{k \ell}=\sum_{i=1}^{n} R_{i k i \ell} \text { and } R_{k \ell, m}=\sum_{i=1}^{n} R_{i k i \ell, m}
$$

Recall that

$$
\Delta_{\hat{g}}:=\sum_{i, j} \hat{g}^{i j} \partial_{y_{i}} \partial_{y_{j}}+\sum_{i, j} \partial_{y_{i}} \hat{g}^{i j} \partial_{y_{j}}+\frac{1}{2} \sum_{i, j} \hat{g}^{i j} \partial_{y_{i}} \log |\hat{g}| \partial_{y_{j}}
$$

A straightforward calculation (still keeping in mind that $v_{0}=\mathcal{O}\left(\epsilon^{2}\right)$ ) shows that

$$
\begin{aligned}
\left(\Delta_{g}-\Delta_{\hat{g}}\right) \phi_{1} & =-\lambda_{1}\left(1-\left(1+v_{0}\right)^{-2}\right) \phi_{1} \\
& +\frac{1}{3} \epsilon^{2} \sum_{i, j, k, \ell} R_{i k j \ell}\left(\frac{y^{i} y^{j} y^{k} y^{\ell}}{r^{2}} \partial_{r}^{2} \phi_{1}+\frac{y^{k} y^{\ell}}{r} \delta_{j}^{i} \partial_{r} \phi_{1}-\frac{y^{i} y^{j} y^{k} y^{\ell}}{r^{3}} \partial_{r} \phi_{1}\right) \\
& -\frac{2}{3} \epsilon^{2} \sum_{i, j} R_{i j} \frac{y^{i} y^{j}}{r} \partial_{r} \phi_{1}+ \\
& +\frac{1}{6} \epsilon^{3} \sum_{i, j, k, \ell, m} R_{i k j \ell, m} \frac{y^{i} y^{j} y^{k} y^{\ell} y^{m}}{r^{2}}\left(\partial_{r}^{2} \phi_{1}-\frac{\partial_{r} \phi_{1}}{r}\right) \\
& +\frac{1}{6} \epsilon^{3} \sum_{k, j, \ell} R_{k j \ell, \cdot} \frac{y^{j} y^{k} y^{\ell}}{r} \partial_{r} \phi_{1}-\frac{1}{4} \epsilon^{3} \sum_{i, \ell, m} R_{i \ell, m} \frac{y^{i} y^{\ell} y^{m}}{r} \partial_{r} \phi_{1}+\mathcal{O}\left(\epsilon^{4}\right),
\end{aligned}
$$

where $r:=|y|$ and

$$
R_{. k j \ell, \cdot}:=\sum_{i=1}^{n} R_{i k j \ell, i}
$$

Observe that we have used the fact that $R(X, X) \equiv 0$ and the symmetries of the curvature tensor for which if either $i=k$ or $j=\ell$ then $R_{i k j \ell, m}=0$.

Observe that, in the expansion of $\left(\Delta_{\dot{g}}-\Delta_{\hat{g}}\right) \phi_{1}$, terms which contain an even number of coordinates, such as $y^{i} y^{j} y^{k} y^{\ell}$ or $y^{i} y^{j}$ etc. do not contribute to the result since, once multiplied 
EXTREMAL DOMAINS FOR THE FIRST EIGENVALUE OF THE LAPLACE-BELTRAMI OPERATOR 13 by $g\left(\nabla \phi_{1}, a\right)$, their average over $S^{n-1}$ is 0 . Therefore, we can write

$$
\begin{aligned}
\int_{\dot{B}_{1}} \stackrel{g}{g}\left(\nabla \phi_{1} a\right)\left(\Delta_{\grave{g}}-\Delta_{\hat{g}}\right) \phi_{1} \mathrm{dvol}_{\grave{g}}= & \epsilon^{3} \int_{\dot{B}_{1}} \partial_{r} \phi_{1} a_{\sigma} \frac{y^{\sigma}}{r} \\
& \left(\frac{1}{6} \sum_{i, j, k, \ell, m} R_{i k j \ell, m} \frac{y^{t} y^{k} y^{l} y^{i} y^{j}}{r^{2}}\left(\partial_{r}^{2} \phi_{1}-\frac{\partial_{r} \phi_{1}}{r}\right)\right. \\
& \left.+\frac{1}{2} \sum_{j, k, \ell}\left(\frac{1}{3} R_{\cdot k j \ell,-}-\frac{1}{2} R_{k j, \ell}\right) \frac{y^{k} y^{\ell} y^{j}}{r} \partial_{r} \phi_{1}\right) \\
+ & \mathcal{O}\left(\epsilon^{4}\right),
\end{aligned}
$$

We make use of the identities in the Appendix to conclude that (15)

$\int_{\dot{B}_{1}} \stackrel{g}{g}\left(\nabla \phi_{1}, a\right)\left(\Delta_{\grave{g}}-\Delta_{\hat{g}}\right) \phi_{1} \mathrm{dvol}_{\grave{g}}=\frac{1}{2 n(n+2)} \epsilon^{3} g(\nabla \operatorname{Scal}(\mathrm{p}), \Theta(a)) \int_{\dot{B}_{1}} r^{2}\left|\partial_{r} \phi_{1}\right|^{2} \mathrm{dvol}_{\grave{g}}+\mathcal{O}\left(\epsilon^{4}\right)$.

The second estimate follows at once from this computation together with the fact that, when $\bar{v} \equiv 0, \hat{\nu}=\left(1+v_{0}\right) \partial_{r}$ as already mentioned and

$$
\stackrel{\circ}{g}\left(\nabla \phi_{1}, a\right)=\partial_{r} \phi_{1}(1) \stackrel{\circ}{g}(a, \cdot),
$$

on $\partial \stackrel{\circ}{B}_{1}$ since this implies that

$$
\left.\int_{S^{n-1}} \stackrel{\circ}{g}(a, \cdot) \hat{g}(\nabla \hat{\phi}, \hat{\nu})\right|_{\partial \dot{B}_{1}} \operatorname{dvol}_{\grave{g}}=\frac{1+v_{0}}{\partial_{r} \phi_{1}(1)} \int_{S^{n-1}} \stackrel{g}{g}\left(\nabla \phi_{1}, a\right) \frac{\partial \hat{\phi}}{\partial r} \operatorname{dvol}_{\grave{g}}
$$

This completes the proof of the result.

Our next task will be to understand the structure of $L_{0}$, the operator obtained by linearizing $F$ with respect to $\bar{v}$ at $\epsilon=0$ and $\bar{v}=0$. We will see that this operator is a first order elliptic operator which does not depend on the point $p$. Also, we will be interested in various properties of the expansion of $F(p, \epsilon, 0)$ in powers of $\epsilon$.

\section{The STRUCture of $L_{0}$}

We keep the notations of the previous section. We claim that, when $\epsilon=0, \bar{g}=\stackrel{\circ}{g}$. Indeed, observe that, if we use coordinates

$$
\bar{X}(y):=\operatorname{Exp}_{p}^{g}\left(\epsilon \sum_{i} y^{i} E_{i}\right)
$$

to parameterize a neighborhood of $p$ in $M$, the coefficients $\bar{g}_{i j}$ of the metric $\bar{X}^{*} \bar{g}=\epsilon^{-2} \bar{X}^{*} g$ can be expanded as

$$
\bar{g}_{i j}(y)=\delta_{i j}+\frac{1}{3} \sum_{k, \ell} R_{i k j \ell} y^{k} y^{\ell} \epsilon^{2}+\frac{1}{6} \sum_{k, \ell, m} R_{i k j l, m} y^{k} y^{\ell} y^{m} \epsilon^{3}+\mathcal{O}\left(\epsilon^{4}\right)
$$


and, when $\epsilon=0$, we conclude that $X^{*} \bar{g}=\stackrel{\circ}{g}$. Therefore, when $\epsilon=0$ we have $\bar{g}=\stackrel{\circ}{g}$ and (10) becomes

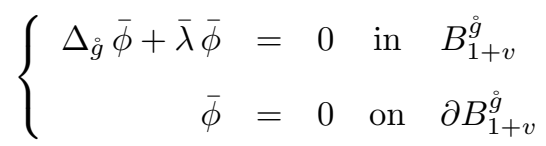

with the normalization

$$
\int_{B_{1+v}^{\grave{g}}} \bar{\phi}^{2} \mathrm{dvol}_{\grave{g}}=1
$$

and the volume constraint

$$
\operatorname{Vol}_{\grave{g}}\left(B_{1+v}^{\stackrel{g}{g}}\right)=\operatorname{Vol}_{\grave{g}}\left(\stackrel{\circ}{B}_{1}\right)
$$

Remember that we have set $v:=v_{0}+\bar{v}$.

We already have established the existence of a unique positive function $\bar{\phi} \in \mathcal{C}^{2, \alpha}\left(S^{n-1}\right)$ (close to $\phi_{1}$ ), a constant $\bar{\lambda} \in \mathbb{R}$ (close to $\lambda_{1}$ ) and a constant $v_{0} \in \mathbb{R}$ (close to 0 ), solutions to the above problem so we are going to construct an expansion of $\bar{\phi}, \bar{\lambda}$ and $v_{0}$ in powers of $\bar{v}$ and its derivatives. This will lead to the structure of the linearized operator $L_{0}$.

Recall that $\lambda_{1}$ is the first eigenvalue of $-\Delta_{g}$ in the unit ball $\stackrel{\circ}{B}_{1}$ with 0 Dirichlet boundary condition and $\phi_{1}$ is the associated eigenfunction which is normalized to be positive and have $L^{2}\left(\stackrel{\circ}{1}_{1}\right)$ norm equal to 1 . Observe that in principle $\phi_{1}$ is only defined in the unit ball, however, this function being radial, it is a solution of a second order ordinary differential equation and as such can be extended at least in a neighborhood of $\partial \stackrel{\circ}{B}_{1}$.

We start with the easy :

Lemma 4.1. Assume that $\bar{v} \in \mathcal{C}_{m}^{2, \alpha}\left(S^{n-1}\right)$ is given. We define

$$
\phi_{0}(x)=\partial_{r} \phi_{1}(x) \bar{v}(x /|x|)
$$

Then

$$
\Delta_{\grave{g}} \phi_{0}+\lambda_{1} \phi_{0}=\frac{1}{r^{2}} \partial_{r} \phi_{1}\left(\Delta_{S^{n-1}}+n-1\right) \bar{v} .
$$

Proof : This is a straightforward exercise. Using the fact that

$$
\Delta_{\grave{g}} \partial_{r} \phi_{1}=-\lambda_{1} \partial_{r} \phi_{1}+\frac{n-1}{r^{2}} \partial_{r} \phi_{1},
$$

we find

$$
\begin{aligned}
\Delta_{\grave{g}} \phi_{0} & =v \Delta_{\grave{g}} \partial_{r} \phi_{1}+\partial_{r} \phi_{1} \Delta_{g} \bar{v}+2 \nabla \bar{v} \nabla \partial_{r} \phi_{1} \\
& =-\lambda_{1} \phi_{0}+\frac{1}{r^{2}} \partial_{r} \phi_{1}\left(\Delta_{S^{n-1}}+n-1\right) \bar{v},
\end{aligned}
$$

This completes the proof of the result. 
For all $\bar{v} \in \mathcal{C}_{m}^{2, \alpha}\left(S^{n-1}\right)$ let $\psi$ be the (unique) solution of

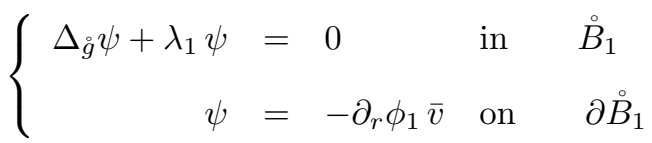

which is $L^{2}\left(\stackrel{\circ}{B}_{1}\right)$-orthogonal to $\phi_{1}$. We define

$$
H(\bar{v}):=\left.\left(\partial_{r} \psi+\partial_{r}^{2} \phi_{1} \bar{v}\right)\right|_{\partial \dot{B}_{1}}
$$

Recall that the eigenvalues of the operator $-\Delta_{S^{n-1}}$ are given by

$$
\mu_{j}=j(n-2+j)
$$

for $j \in \mathbb{N}$. The corresponding eigenspaces will be denoted by $V_{j}$.

We will need the following result :

Proposition 4.2. The operator

$$
H: \mathcal{C}_{m}^{2, \alpha}\left(S^{n-1}\right) \longrightarrow \mathcal{C}_{m}^{1, \alpha}\left(S^{n-1}\right),
$$

is a self adjoint, first order elliptic operator. (Recall that the subscript $m$ is meant to point out that functions have mean 0 on $\left.S^{n-1}\right)$. The kernel of $H$ is given by $V_{1}$, the eigenspace of $-\Delta_{S^{n-1}}$ associated to the eigenvalue $n-1$. Moreover there exists $c>0$ such that

$$
\|w\|_{\mathcal{C}^{2, \alpha}\left(S^{n-1}\right)} \leq c\|H(w)\|_{\mathcal{C}^{1, \alpha}\left(S^{n-1}\right)},
$$

provided $w$ is $L^{2}\left(S^{n-1}\right)$-orthogonal to $V_{0} \oplus V_{1}$.

Proof : The fact that $H$ is a first order elliptic operator is standard since it is the sum of the Dirichlet-to-Neumann operator for $\Delta_{\mathscr{g}}+\lambda_{1}$ and a constant times the identity. In particular, elliptic estimates yield

$$
\|H(w)\|_{\mathcal{C}^{1, \alpha}\left(S^{n-1}\right)} \leq c\|w\|_{\mathcal{C}^{2, \alpha}\left(S^{n-1}\right)}
$$

The fact that the operator $H$ is (formally) self-adjoint is easy. Let $\psi_{1}$ (resp. $\psi_{2}$ ) the solution of (20) corresponding to the function $w_{1}$ (resp. $w_{2}$ ). We compute

$$
\begin{aligned}
\partial_{r} \phi_{1}(1) \int_{\partial \dot{B}_{1}}\left(H\left(w_{1}\right) w_{2}-w_{1} H\left(w_{2}\right)\right) \mathrm{dvol}_{\grave{g}} & =\partial_{r} \phi_{1}(1) \int_{\partial \dot{B}_{1}}\left(\partial_{r} \psi_{1} w_{2}-\partial_{r} \psi_{2} w_{1}\right) \mathrm{dvol}_{\grave{g}} \\
& =\int_{\partial_{\dot{B}_{1}}}\left(\psi_{1} \partial_{r} \psi_{2}-\psi_{2} \partial_{r} \psi_{1}\right) \mathrm{dvol}_{\grave{g}} \\
& =\int_{\dot{B}_{1}}\left(\psi_{1} \Delta_{\grave{g}} \psi_{2}-\psi_{2} \Delta_{\grave{g}} \psi_{1}\right) \mathrm{dvol}_{\grave{g}} \\
& =0
\end{aligned}
$$

To prove the other statements, we define for all $w \in \mathcal{C}_{m}^{2, \alpha}\left(S^{n-1}\right), \Psi$ to be the continuous solution of

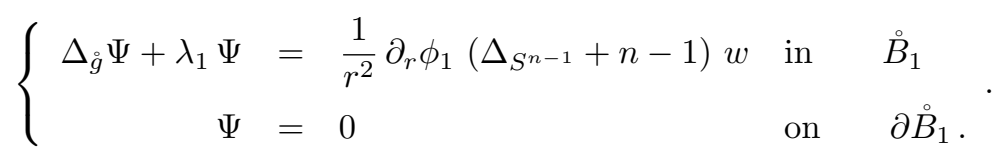


Observe that $\partial_{r} \phi_{1}$ vanishes at first order at $r=0$ and hence the right hand side is bounded by a constant times $r^{-1}$ near the origin. Standard elliptic estimates then imply that the solution $\Psi$ is at least continuous near the origin. A straightforward computation using the result of Lemma 4.1 and writing $\Psi=\psi+\partial_{r} \phi_{1} w$, shows that

$$
H(w):=\left.\partial_{r} \Psi\right|_{\partial \check{B}_{1}}
$$

With this alternative definition, it should be clear that $H$ preserves the eigenspaces $V_{j}$ and in particular, $H$ maps into the space of functions whose mean over $S^{n-1}$ is 0 . Moreover, it is clear that $V_{1}$ is included in the kernel of $H$ since $\left(\Delta_{S^{n-1}}+n-1\right) w=0$ for any $w \in V_{1}$. We now prove that $V_{1}$ is the only kernel of this operator.

We consider

$$
w=\sum_{j \geq 1} w_{j}
$$

the eigenfunction decomposition of $w$. Namely $w_{j} \in V_{j}$. Then

$$
H(w)=\sum_{j} \alpha_{j} w_{j}
$$

where the constants $\alpha_{j}$ are given by

$$
\alpha_{j}=\partial_{r} a_{j}(1)
$$

where $a_{j}$ is the continuous solution of

$$
a_{j}^{\prime \prime}+\frac{n-1}{r} a_{j}^{\prime}+\lambda_{1} a_{j}-\frac{1}{r^{2}} \mu_{j} a_{j}=\frac{1}{r^{2}}\left(n-1-\mu_{j}\right) \partial_{r} \phi_{1},
$$

with $a_{j}(1)=0$.

Observe that $\alpha_{1}=0$ and, in order to prove that the kernel of $H$ is given by $V_{1}$, it is enough to show that $\alpha_{j} \neq 0$ for all $j \geq 2$.

We claim that

$$
a_{j} \leq 0,
$$

for all $j \geq 2$. This follows at once from the maximum principle since $n-1-\mu_{j}<0$ for all $j \geq 2$ and $\partial_{r} \phi_{1} \leq 0$.

Proof of the claim : By definition of $\lambda_{1}$, the operator $\Delta_{g}+\lambda_{1}$ is non-positive, in the sense that

$$
-\int_{\dot{B}_{1}} u\left(\Delta_{\grave{g}}+\lambda_{1}\right) u \mathrm{dvol}_{\grave{g}}=\int_{\dot{B}_{1}}\left(|\nabla u|_{\grave{g}}^{2}-\lambda_{1} u^{2}\right) \operatorname{dvol}_{\grave{g}} \geq 0 .
$$

Specializing this inequality to radial functions, we get

$$
\int_{0}^{1}\left(\left(\partial_{r} u\right)^{2}-\lambda_{1} u^{2}\right) r^{n-1} \mathrm{dr} \geq 0
$$

provided $u \in H_{0}^{1}\left(\stackrel{\circ}{B}_{1}\right)$ is radial. 
EXTREMAL DOMAINS FOR THE FIRST EIGENVALUE OF THE LAPLACE-BELTRAMI OPERATOR 17

Now, assume that $a_{j} \geq 0$ in $\left[r_{1}, r_{2}\right]$ with $a_{j}\left(r_{i}\right)=0$, then multiplying (24) by $a_{j} r^{n-1}$ and integrating the result by parts between $r_{1}$ and $r_{2}$, we get

$$
\int_{r_{1}}^{r_{2}}\left(\left(\partial_{r} a_{j}\right)^{2}-\lambda_{1} a_{j}^{2}+\frac{1}{r^{2}} \mu_{j} a_{j}^{2}\right) r^{n-1} \mathrm{dr} \leq 0
$$

and hence necessarily $a_{j} \equiv 0$ on $\left[r_{1}, r_{2}\right]$. This completes the proof of the claim.

The claim being proven, we use the fact that $a_{j}(1)=0$ for all $j \geq 2$ to conclude that

$$
0 \leq \partial_{r} a_{j}(1) \text {. }
$$

If $\partial_{r} a_{j}(1)=0$ then necessarily $\partial_{r}^{2} a_{j}(1) \leq 0$ but evaluation of $(24)$ at $r=1$ implies that

$$
\begin{aligned}
0=(n-1) a_{j}^{\prime}(1) & =\left(n-1-\mu_{j}\right) \partial_{r} \phi_{1}(1)-a_{j}^{\prime \prime}(1) \\
& \geq\left(n-1-\mu_{j}\right) \partial_{r} \phi_{1}(1) \\
& >0,
\end{aligned}
$$

which immediately leads to a contradiction. Hence, $\partial_{r} a_{j}(1)>0$ for all $j \geq 2$ and this completes the proof of the fact that the kernel of the operator $H$ is equal to $V_{1}$.

The main result of this section is the following :

Proposition 4.3. The operator $L_{0}$ is equal to $H$.

Proof : By definition, the operator $L_{0}$ is the linear operator obtained by linearizing $N$ with respect to $\bar{v}$ at $\epsilon=0$ and $\bar{v}=0$. In other words, we have

$$
L_{0}(\bar{w})=\lim _{s \rightarrow 0} \frac{F(p, 0, s \bar{w})-F(p, 0,0)}{s} .
$$

Since $\epsilon=0$, we have already seen that $\bar{g}=\stackrel{\circ}{g}$. Writing $\bar{v}=s \bar{w}$, we argue as in the proof of Proposition 3.2 and consider the parameterization of $\stackrel{\circ}{B}_{1+v}$ given by

$$
Y(y):=\left(1+v_{0}+s \chi(y) \bar{w}\left(\frac{y}{|y|}\right)\right) y
$$

where $\chi$ is a cutoff function identically equal to 0 when $|y| \leq 1 / 2$ and identically equal to 1 when $|y| \geq 3 / 4$. We set

$$
\hat{g}:=Y^{*} \stackrel{\circ}{g}
$$

so that $\hat{\phi}=Y^{*} \bar{\phi}, \hat{\lambda}=\bar{\lambda}$ and $v_{0}$ are solutions (smoothly depending on the real parameter $s$ ) of

with

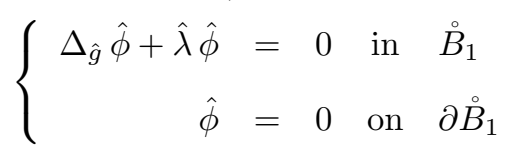

and

$$
\int_{\dot{B}_{1}} \hat{\phi}^{2} \operatorname{dvol}_{\hat{g}}=1
$$

$$
\operatorname{Vol}_{\hat{g}}\left(\stackrel{\circ}{B}_{1}\right)=\operatorname{Vol}_{\grave{g}}\left(\stackrel{\circ}{B}_{1}\right)
$$


We remark that $\hat{\phi}_{1}:=Y^{*} \phi_{1}$ is a solution of

$$
\Delta_{\hat{g}} \hat{\phi}_{1}+\lambda_{1} \hat{\phi}_{1}=0
$$

since $\hat{g}=Y^{*} \stackrel{\circ}{g}$. Moreover

$$
\hat{\phi}_{1}(y)=\phi_{1}\left(\left(1+v_{0}+s \bar{w}(y)\right) y\right),
$$

on $\partial \dot{B}_{1}$. Writing $\hat{\phi}=\hat{\phi}_{1}+\hat{\psi}$ and $\hat{\lambda}=\lambda_{1}+\mu$, we find that

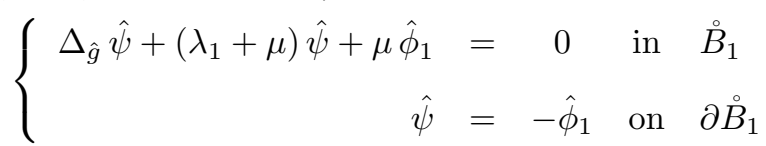

with

$$
\int_{\stackrel{\circ}{1}_{1}}\left(2 \hat{\phi}_{1} \hat{\psi}+\hat{\psi}^{2}\right) \operatorname{dvol}_{\hat{g}}=\int_{\dot{B}_{1}} \phi_{1}^{2} \operatorname{dvol}_{\grave{g}}-\int_{\dot{B}_{1+v_{0}+s \bar{w}}} \phi_{1}^{2} \operatorname{dvol}_{\grave{g}}
$$

and

$$
\operatorname{Vol}_{\hat{g}}\left(\stackrel{\circ}{B}_{1}\right)=\operatorname{Vol}_{\grave{g}}\left(\stackrel{\circ}{B}_{1}\right)
$$

Obviously $\hat{\psi}, \mu$ and $v_{0}$ are smooth functions of $s$. When $s=0$, we have $\bar{\phi}=\phi_{1}, \bar{\lambda}=\lambda_{1}$ and $v_{0}=0$. Therefore, $\hat{\psi}, \mu$ and $v_{0}$ all vanish and $\hat{\phi}_{1}=\phi_{1}$, when $s=0$. Moreover $\hat{g}=\stackrel{g}{g}$ when $s=0$. We set

$$
\dot{\psi}=\left.\partial_{s} \hat{\psi}\right|_{s=0}, \quad \dot{\mu}=\left.\partial_{s} \mu\right|_{s=0}, \quad \text { and } \quad \dot{v}_{0}=\left.\partial_{s} v_{0}\right|_{s=0},
$$

Differentiating (26) with respect to $s$ and evaluating the result at $s=0$, we obtain

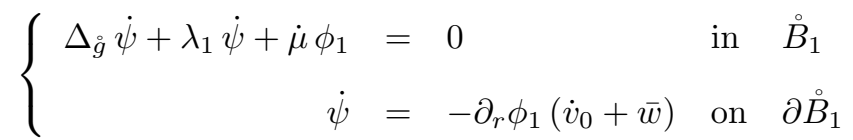

Observe that, as already mentioned, $\hat{\phi}_{1}(y)=\phi_{1}\left(\left(1+v_{0}+s \bar{w}(y)\right) y\right)$ on $\partial \stackrel{\circ}{B}_{1}$ and differentiation with respect to $s$ at $s=0$ yields $\left.\partial_{s} \hat{\phi}_{1}\right|_{s=0}=\partial_{r} \phi_{1}\left(\dot{v}_{0}+\bar{w}\right)$.

Differentiating (27) with respect to $s$ and evaluating the result at $s=0$, we obtain

$$
\int_{\dot{B}_{1}} \phi_{1} \dot{\psi} \mathrm{dvol}_{\stackrel{g}{g}}=0
$$

Indeed, the derivative of the right hand side of (27) with respect to $s$ vanishes when $s=0$ since $\phi_{1}$ vanishes identically on $\partial \stackrel{\circ}{B}_{1}$.

Finally, differentiating (28) with respect to $s$ and evaluating the result at $s=0$, we obtain

$$
\int_{S^{n-1}}\left(\dot{v}_{0}+\bar{w}\right) \operatorname{dvol}_{\grave{g}}=0
$$

The last equality immediately implies (since, by definition, the average of $\bar{w}$ is 0 ) that $\dot{v}_{0}=0$. If we multiply the first equation of (29) by $\phi_{1}$ and we integrate it, using the boundary condition and the fact that the average of $\bar{w}$ is 0 together with the fact that $\dot{v}_{0}=0$, we conclude that $\dot{\mu}=0$. And hence $\dot{\psi}$ is precisely the solution of (20). To summarize, we have proven that

$$
\hat{\phi}=\hat{\phi}_{1}+s \psi+\mathcal{O}\left(s^{2}\right)
$$


where $\psi$ is the solution of $(20)$ and we also know that

$$
v_{0}=\mathcal{O}\left(s^{2}\right)
$$

In particular, in $\stackrel{\circ}{B}_{1} \backslash \stackrel{\circ}{B}_{3 / 4}$, we have

$$
\begin{aligned}
\hat{\phi}(y) & =\phi_{1}((1+s \bar{w}(y /|y|)) y)+s \psi(y)+\mathcal{O}\left(s^{2}\right) \\
& =\phi_{1}(y)+s\left(\bar{w}(y /|y|) r \partial_{r} \phi_{1}+\psi\right)+\mathcal{O}\left(s^{2}\right)
\end{aligned}
$$

where we have set $r:=|y|$.

To complete the proof of the result, it suffices to compute the normal derivative of the function $\hat{\phi}$ when the normal is computed with respect to the metric $\hat{g}$. We use polar coordinates $y=r z$ where $r>0$ and $z \in S^{n-1}$. Then the metric $\hat{g}$ can be expanded in $\stackrel{\circ}{B}_{1} \backslash \stackrel{\circ}{B}_{3 / 4}$ as

$$
\hat{g}=\left(1+v_{0}+s \bar{w}\right)^{2} d r^{2}+2 s\left(1+v_{0}+s \bar{w}\right) r d \bar{w} d r+r^{2}\left(1+v_{0}+s \bar{w}\right)^{2} \stackrel{\circ}{h}+s^{2} r^{2} d \bar{w}^{2}
$$

where $h$ is the metric on $S^{n-1}$ induced by the Euclidean metric. It follows from this expression together with the fact that $v_{0}=\mathcal{O}\left(s^{2}\right)$ that the unit normal vector field to $\partial \stackrel{\circ}{B}_{1}$ for the metric $\hat{g}$ is given by

$$
\hat{\nu}=\left((1+s \bar{w})^{-1}+\mathcal{O}\left(s^{2}\right)\right) \partial_{r}+\mathcal{O}(s) \partial_{z_{j}}
$$

where $\partial_{z_{j}}$ are vector fields induced by a parameterization of $S^{n-1}$. Using this, we conclude that

$$
\hat{g}\left(\nabla \hat{\phi}_{1}, \hat{\nu}\right)=\partial_{r} \phi_{1}+s\left(\bar{w} \partial_{r}^{2} \phi_{1}+\partial_{r} \psi\right)+\mathcal{O}\left(s^{2}\right)
$$

on $\partial \stackrel{\circ}{B}_{1}$. The result then follows at once from the fact that $\partial_{r} \phi_{1}$ is constant while the term $\bar{w} \partial_{r}^{2} \phi_{1}+\partial_{r} \psi$ has mean 0 on the boundary $\partial \stackrel{\circ}{B_{1}}$. This completes the proof of the proposition.

We denote by $L_{\epsilon}$ the linearization of $F$ with respect to $\bar{v}$, computed at the point $(p, \epsilon, 0)$. Following the proof of the previous Proposition, it is easy to check the :

Lemma 4.4. There exists a constant $c>0$ such that, for all $\epsilon>0$ small enough we have the estimate

$$
\left\|\left(L_{\epsilon}-L_{0}\right) \bar{v}\right\|_{\mathcal{C}^{1, \alpha}} \leq c \epsilon^{2}\|\bar{v}\|_{\mathcal{C}^{2, \alpha}}
$$

Proof : Clearly both $L_{\epsilon}$ and $L_{0}$ are first order differential operators. To prove the estimate, we simply use the fact that, when $\epsilon \neq 0$, the difference between the coefficients of $\bar{g}=\epsilon^{-2} g$ and $\stackrel{\circ}{g}$ can be estimated by a constant times $\epsilon^{2}$. This implies that the discrepancy between the linearized operator when $\epsilon=0$ and when $\epsilon \neq 0$ is a first order differential operator whose coefficients can be estimated by a constant times $\epsilon^{2}$.

The main result of this section is the fact that the linearized operator $L_{0}$ is given by $H$. Observe that the kernel of $L_{0}$ is equal to $V_{1}$ which is the vector space spanned by the restriction of linear functions to the unit sphere. This is geometrically very natural since, when $\epsilon=0$, a linear function $\bar{v}:=\stackrel{\circ}{g}(a, \cdot) \in V_{1}$ correspond to infinitesimal translation of the unit ball in the direction $a \in \mathbb{R}^{n}$. Therefore we have

$$
B_{1+s \bar{v}}^{\stackrel{\circ}{g}}(p) \sim \stackrel{\circ}{B}_{1}(p+s a)
$$


This implies that the solution of (8) is given by $\phi_{1}$ (modulo some $\mathcal{O}\left(s^{2}\right)$ term) and hence its normal data is constant (modulo some $\mathcal{O}\left(s^{2}\right)$ term). Therefore $F(p, 0, \bar{v})=\mathcal{O}\left(s^{2}\right)$ which shows that $L_{0} \bar{v}=0$.

\section{The PRoOf of Theorem 1.3}

We shall now prove that, for $\epsilon>0$ small enough, it is possible to solve the equation

$$
F(p, \epsilon, \bar{v})=0
$$

Unfortunately, we will not be able to solve this equation at once. Instead, we first prove the :

Proposition 5.1. There exists $\epsilon_{0}>0$ such that, for all $\epsilon \in\left[0, \epsilon_{0}\right]$ and for all $p \in M$, there exists a unique function $\bar{v}=\bar{v}(p, \epsilon)$ and a vector $a=a(p, \epsilon) \in \mathbb{R}^{n}$ such that

$$
F(p, \epsilon, \bar{v})+\stackrel{\circ}{g}(a, \cdot)=0
$$

The function $\bar{v}$ and the vector a depend smoothly on $p$ and $\epsilon$ and we have

$$
|a|+\|\bar{v}\|_{\mathcal{C}^{2, \alpha}\left(S^{n-1}\right)} \leq c \epsilon^{2}
$$

Proof : We fix $p \in M$ and define

$$
\bar{F}(p, \epsilon, \bar{v}, a):=F(p, \epsilon, \bar{v})+\stackrel{\circ}{g}(a, \cdot)
$$

It is easy to check that $\bar{F}$ is a smooth map from a neighborhood of $(p, 0,0,0)$ in $M \times[0, \infty) \times$ $\mathcal{C}_{m}^{2, \alpha}\left(S^{n-1}\right) \times \mathbb{R}^{n}$ into a neighborhood of 0 in $\mathcal{C}^{1, \alpha}\left(S^{n-1}\right)$. Moreover,

$$
\bar{F}(p, 0,0,0)=0
$$

and the differential of $\bar{F}$ with respect to $\bar{v}$, computed at $(p, 0,0,0)$ is given by $H$. Finally the image of the linear map $a \longmapsto \stackrel{g}{g}(a, \cdot)$ is just the vector space $V_{1}$. Thanks to the result of Proposition 4.2, the implicit function theorem applies to get the existence of $\bar{v}$ and $a$, smoothly depending on $p$ and $\epsilon$ such that $F(p, \epsilon, \bar{v})+\stackrel{\circ}{g}(a, \cdot)=0$. The estimate for $\bar{v}$ and $a$ follows at once from Lemma 3.3.

In view of the result of the previous Proposition, it is enough to show that, provided that $\epsilon$ is small enough, it is possible to choose the point $p \in M$ such that $a(p, \epsilon)=0$. We claim that, there exists a constant $\tilde{C}>0$ (only depending on $n$ ) such that

$$
\Theta(a(p, \epsilon))=-\epsilon^{3} \tilde{C} \nabla^{g} \operatorname{Scal}(p)+\mathcal{O}\left(\epsilon^{4}\right)
$$

Indeed, for all $b \in \mathbb{R}^{n}$ we compute

$$
\begin{aligned}
& \int_{S^{n-1}} \stackrel{\circ}{g}(a, \cdot) \stackrel{\circ}{g}(b, \cdot) \mathrm{dvol}_{\grave{g}}=-\int_{S^{n-1}} F(p, \epsilon, \bar{v}) \stackrel{\circ}{g}(b, \cdot) \mathrm{dvol}_{\grave{g}} \\
& =-\int_{S^{n-1}}\left(F(p, \epsilon, 0)+L_{0} \bar{v}\right) \stackrel{\circ}{g}(b, \cdot) \operatorname{dvol}_{g} \\
& -\int_{S^{n-1}}\left(F(p, \epsilon, \bar{v})-F(p, \epsilon, 0)-L_{\epsilon} \bar{v}\right) \stackrel{\circ}{g}(b, \cdot) \operatorname{dvol}_{g} \\
& -\int_{S^{n-1}}\left(L_{\epsilon}-L_{0}\right) \bar{v} \stackrel{\circ}{g}(b, \cdot) \mathrm{dvol}_{g}
\end{aligned}
$$


EXTREMAL DOMAINS FOR THE FIRST EIGENVALUE OF THE LAPLACE-BELTRAMI OPERATOR 21

Now, we use the fact that $\bar{v}$ is $L^{2}\left(S^{n-1}\right)$-orthogonal to linear functions and hence so is $L_{0} \bar{v}$. Therefore,

$$
\int_{S^{n-1}} L_{0} \bar{v} \stackrel{\circ}{g}(b, \cdot) \mathrm{dvol}_{\grave{g}}=0
$$

Using the fact that $\bar{v}=\mathcal{O}\left(\epsilon^{2}\right)$, we get

$$
F(p, \epsilon, \bar{v})-F(p, \epsilon, 0)-L_{\epsilon} \bar{v}=\mathcal{O}\left(\epsilon^{4}\right)
$$

Similarly, it follows from the result of Lemma 4.4 that

$$
\left(L_{\epsilon}-L_{0}\right) \bar{v}=\mathcal{O}\left(\epsilon^{4}\right)
$$

The claim then follows from the second estimate in Lemma 3.3 and the fact that

$$
\int_{S^{n-1}} \stackrel{g}{g}(a, \cdot) \stackrel{\circ}{g}(b, \cdot) \mathrm{dvol}_{\grave{g}}=g(\Theta(a), \Theta(b)) \int_{S^{n-1}}\left(x_{1}\right)^{2} \operatorname{dvol}_{\grave{g}}=\frac{1}{n} \operatorname{Vol}_{\grave{g}}\left(S^{n-1}\right) g(\Theta(a), \Theta(b)) .
$$

Now if we assume that $p_{0}$ is a nondegenerate critical point of the scalar curvature function, we can apply once more the implicit function theorem to solve the equation

$$
G(\epsilon, p):=\epsilon^{-3} \Theta(a(p, \epsilon))=0 .
$$

It should be clear that $G$ depends smoothly on $\epsilon \in\left[0, \epsilon_{0}\right)$ and $p \in M$. Moreover, we have

$$
G(0, p)=-\tilde{C} \nabla^{g} \operatorname{Scal}(p)
$$

and hence $G\left(0, p_{0}\right)=0$. By assumption the differential of $G$ with respect to $p$, computed at $p_{0}$ is invertible. Therefore, for all $\epsilon$ small enough there exists $p_{\epsilon}$ close to $p_{0}$ such that

$$
\Theta\left(a\left(p_{\epsilon}, \epsilon\right)\right)=0
$$

In addition we have

$$
\operatorname{dist}\left(p_{0}, p_{\epsilon}\right) \leq c \epsilon
$$

This completes the proof the Theorem 1.3.

\section{Appendix}

Lemma 6.1. For all $\sigma=1, \ldots, n$, we have

$$
\sum_{i, j, k, \ell, m} \int_{S^{n-1}} R_{i k j \ell, m} x^{i} x^{j} x^{k} x^{\ell} x^{m} x^{\sigma} \operatorname{dvol}_{\grave{g}}=0 .
$$

Proof : To see that we consider all terms of the above sum, obtained fixing the 6-tuple $(i, k, j, \ell, m, \sigma)$. We observe that if in such a 6 -tuple there is an element that appears an odd number of time then $\int_{S^{n-1}} x^{i} x^{j} x^{k} x^{\ell} x^{m} x^{\sigma}{\mathrm{dvol}_{g}}_{g}=0$. Moreover, the symmetries of the curvature tensor imply that, if either $i=k$ or $j=\ell$, then $R_{i k j \ell, m}=0$. Therefore, we have to compute

$$
\sum_{i, k, \sigma} \int_{S^{n-1}} R^{*}\left(x^{i}\right)^{2}\left(x^{k}\right)^{2}\left(x^{\sigma}\right)^{2} \operatorname{dvol}_{\grave{g}}
$$


where

$R^{*}:=R_{i k i k, \sigma}+R_{i k i \sigma, k}+R_{i k k i, \sigma}+R_{i k \sigma i, k}+R_{i k k \sigma, i}+R_{i k \sigma k, i}+R_{i \sigma i k, k}+R_{\sigma k i k, i}+R_{i \sigma k i, k}+R_{\sigma k k i, i}$ Again, we apply the symmetries of Riemann curvature which imply that $R_{i k i k, \sigma}+R_{i k k i, \sigma}=0$, $R_{i k i \sigma, k}+R_{i k \sigma i, k}=0, R_{i k k \sigma, i}+R_{i k \sigma k, i}=0, R_{\sigma k i k, i}+R_{\sigma k k i, i}=0$ and $R_{i \sigma i k, k}+R_{i \sigma k i, k}=0$, and we conclude that the sum is equal to 0 .

Lemma 6.2. For all $\sigma=1, \ldots, n$, we have

$$
\sum_{j, k, \ell} \int_{S^{n-1}} R_{\cdot k j \ell, \cdot} x^{j} x^{k} x^{\ell} x^{\sigma} \mathrm{dvol}_{g}=0 .
$$

Proof : Arguing as in the previous proof, we find that $\int_{S^{n-1}} x^{j} x^{k} x^{\ell} x^{\sigma} \mathrm{dvol}_{\grave{g}}=0$ unless the indices $j, k, \ell, \sigma$ are pairwise equal. Hence, we can write

$$
\begin{aligned}
\sum_{j, k, \ell} \int_{S^{n-1}} R_{\cdot k j \ell, \cdot} x^{j} x^{k} x^{\ell} x^{\sigma} \mathrm{dvol}_{\grave{g}} & =\int_{S^{n-1}} R_{\cdot \sigma \sigma \sigma, \cdot}\left(x^{\sigma}\right)^{4} \mathrm{dvol}_{\grave{g}} \\
& +\sum_{j \neq \sigma} \int_{S^{n-1}}\left(R_{\cdot \sigma j j, \cdot}+R_{\cdot j \sigma j, \cdot}+R_{\cdot j j \sigma, \cdot}\right)\left(x^{\sigma}\right)^{2}\left(x^{j}\right)^{2} \mathrm{dvol}_{\grave{g}}
\end{aligned}
$$

Using the symmetries of the Riemann curvature tensor, we get $R_{\cdot \sigma \sigma \sigma, \cdot}=R_{\cdot \sigma j j, \cdot}=0$ and $R_{\cdot j \sigma j, .}+$ $R_{. j j \sigma, .}=0$. This completes the proof of the result.

Lemma 6.3. For all $\sigma=1, \ldots, n$, we have

$$
\sum_{i, \ell, m} \int_{S^{n-1}} R_{i \ell, m} x^{i} x^{\ell} x^{m} x^{\sigma} \operatorname{dvol}_{\grave{g}}=\frac{2}{n(n+2)} \operatorname{Vol}_{\grave{g}}\left(S^{n-1}\right) \mathrm{Scal}_{, \sigma}
$$

Proof : Again, we find that $\int_{S^{n-1}} x^{i} x^{\ell} x^{m} x^{\sigma} \mathrm{dvol}_{g}=0$ unless the indices $i, \ell, m, \sigma$ are pairwise equal. Hence we can write

$$
\begin{aligned}
\sum_{i, \ell, m} \int_{S^{n-1}} R_{i \ell, m} x^{i} x^{\ell} x^{m} x^{\sigma} \mathrm{dvol}_{\grave{g}}= & R_{\sigma \sigma, \sigma} \int_{S^{n-1}}\left(x^{\sigma}\right)^{4} \mathrm{dvol}_{\grave{g}} \\
& +\sum_{j \neq \sigma} \int_{S^{n-1}}\left(R_{\sigma j, j}+R_{j \sigma, j}+R_{j j, \sigma}\right)\left(x^{\sigma}\right)^{2}\left(x^{j}\right)^{2} \mathrm{dvol}_{\grave{g}} \\
= & R_{\sigma \sigma, \sigma} \int_{S^{n-1}}\left(x^{1}\right)^{4} \mathrm{dvol}_{\grave{g}} \\
& +\sum_{j \neq \sigma}\left(R_{\sigma j, j}+R_{j \sigma, j}+R_{j j, \sigma}\right) \int_{S^{n-1}}\left(x^{1}\right)^{2}\left(x^{2}\right)^{2} \mathrm{dvol}_{\grave{g}} \\
= & R_{\sigma \sigma, \sigma}\left(\int_{S^{n-1}}\left(x^{1}\right)^{4} \operatorname{dvol}_{\grave{g}}-3 \int_{S^{n-1}}\left(x^{1}\right)^{2}\left(x^{2}\right)^{2} \mathrm{dvol}_{\grave{g}}\right) \\
& +\sum_{j}\left(R_{\sigma j, j}+R_{j \sigma, j}+R_{j j, \sigma}\right) \int_{S^{n-1}}\left(x^{1}\right)^{2}\left(x^{2}\right)^{2} \mathrm{dvol}_{\grave{g}}
\end{aligned}
$$


Now we use the fact that

$$
\int_{S^{n-1}}\left(x^{1}\right)^{4} \mathrm{dvol}_{\grave{g}}=3 \int_{S^{n-1}}\left(x^{1}\right)^{2}\left(x^{2}\right)^{2} \mathrm{dvol}_{\grave{g}}=\frac{3}{n(n+2)} \operatorname{Vol}_{\grave{g}}\left(S^{n-1}\right),
$$

to conclude that

$$
\sum_{i, \ell, m} \int_{S^{n-1}} R_{i \ell, m} x^{i} x^{\ell} x^{m} x^{\sigma} \operatorname{dvol}_{\grave{g}}=\frac{1}{n(n+2)} \operatorname{Vol}_{\grave{g}}\left(S^{n-1}\right) \sum_{j}\left(R_{\sigma j, j}+R_{j \sigma, j}+R_{j j, \sigma}\right)
$$

Finally, the second Bianchi identity yields

$$
\sum_{j} R_{\sigma j, j}=\sum_{j} R_{j \sigma, j}=\frac{1}{2} \mathrm{Scal}_{, \sigma}
$$

and by definition $\sum_{j} R_{j j, \sigma}=\mathrm{Scal}_{, \sigma}$. Hence

$$
\sum_{i, \ell, m} \int_{S^{n-1}} R_{i \ell, m} x^{i} x^{\ell} x^{m} x^{\sigma} \operatorname{dvol}_{g}=\frac{2}{n(n+2)} \operatorname{Vol}_{\grave{g}}\left(S^{n-1}\right) \operatorname{Scal}_{, \sigma}
$$

This completes the proof of the result.

\section{REFERENCES}

[1] O. Druet. Sharp local isoperimetric inequalities involving the scalar curvature. Proc. Amer. Math. Soc. 130 (2002), no. 8, 2351-2361.

[2] A. El Soufi and S. Ilias, Domain deformations and eigenvalues of the Dirichlet Laplacian in Riemannian manifold, Illinois Journal of Mathematics 51 (2007) 645-666.

[3] P. R. Garadedian and M. Schiffer. Variational problems in the theory of elliptic partial differetial equations, Journal of Rational Mechanics and Analysis 2 (1953), 137-171.

[4] G. Faber. Beweis dass unter allen homogenen Menbranen von gleicher Fläche und gleicher Spannung die kreisförmige den tiefsten Grundtonggibt, Sitzungsber. Bayer. Akad. der Wiss. Math.-Phys. , p.169-172, Munich, 1923.

[5] D. Gilbarg and N. S. Trudinger. Elliptic Partial Differential Equations of Second Order, Grundlehren der mathematischen Wissenschaften, a Series of Comprehensive Studies in Mathematics, Vol. 224, $2^{\text {nd }}$ Edition, Springer 1977, 1983.

[6] E. Krahn. Uber eine von Raleigh formulierte Minimaleigenschaft der Kreise, Math. Ann., 94 (1924), 97100.

[7] E. Krahn. Uber Minimaleigenschaften der Kugel in drei und mehr dimensionen, Acta Comm. Univ. Tartu (Dorpat), A9 (1926), 144.

[8] J. M. Lee and T. H. Parker. The Yamabe Problem, Bulletin of the American Mathematical Society 17, 1 (1987), 37-91.

[9] S. Nardulli. Le profil isopérimétrique d'une variété riemannienne compacte pour les petits volumes, Thèse de l'Université Paris 11 (2006).

[10] F. Pacard and X. Xu. Constant mean curvature sphere in riemannian manifolds, preprint.

[11] R. Schoen and S. T. Yau. Lectures on Differential Geometry, International Press (1994).

[12] T. J. Willmore. Riemannian Geometry, Oxford Science Publications (1996).

[13] R. Ye. Foliation by constant mean curvature spheres, Pacific Journal of Mathematics, Vol.147 n.2 (1991), 381-396.

[14] D. Z. Zanger. Eigenvalue variation for the Neumann problem, Applied Mathematics Letters 14 (2001), 39-43. PaCARd@Univ-Paris12.Fr, Université Paris-Est and Institut Universitaire de France, France Pieralberto.sicbaldi@univ-PARis12.Fr, Université Paris-Est, France 